\title{
NONCONGRUENT MINIMAL SURFACES WITH THE SAME SYMMETRIES AND CONFORMAL STRUCTURE
}

\author{
VALÉRIO RAMOS BATISTA
}

(Received September 24, 2002, revised June 3, 2003)

\begin{abstract}
Concerning complete orientable minimal surfaces with finite total curvature in Euclidean three-space, we show for any positive genus the existence of noncongruent examples having the same symmetry group and conformal type.
\end{abstract}

1. Introduction. Since 1761, when Langrange first published the "Differential Equation of the Minimal Surfaces", until the first half of the twentieth century, the theory of these surfaces had a slow development but had important contributions made, for instance, by Scherk (1835), Enneper, Weierstrass (1882), Schwarz (1890) and Riemann (1898). In the case of complete minimal surfaces in Euclidean three-space $\boldsymbol{R}^{3}$, after Riemann's work more than a half century had passed until Huber (1957) and Osserman (1964) introduced new important results (see [7] and [13]). With these, the theory of minimal surfaces gained a new impulse that still motivates the research in this area.

One main research goal in the theory is the classification of complete minimal surfaces in $\boldsymbol{R}^{3}$. All possible examples of such surfaces have been classified under certain special restrictions on the conformal type, ends, symmetry group, periodicity and/or total curvature. In the case of embedded examples with finite total curvature, Schoen's [17] and López-Ros' [11] theorems classify the cases of one end (plane) and two ends (catenoid). For any embedded example with three ends and genus one, Costa proved that it must be his own surface or its deformations found by Hoffman and Karcher (see [3] and [4]). It is not known if there exists an embedded example with four ends and genus one, while there is an example with four ends and genus two. Thus the question of a general classification of embedded minimal surfaces is still wide open.

Even if one forfeits embeddedness, the finite total curvature case still presents difficulties. For instance, the symmetry group, together with the conformal type, does not characterize minimal surfaces of genus zero (see [9]). For positive genus, however, having both equal symmetry group and equal conformal type excludes several close cases, like Costa's [2] and Chen-Gackstatter's [1] examples. They have the same symmetry group but only the "almost" same conformal type. This difficulty of finding some coincidence is explained by the fact that, for each Weierstrass pair on a family of compact Riemann surfaces, the closure of the period problems commonly succeeds for at most one choice of the parameters for each member of

2000 Mathematics Subject Classification. Primary 53A10; Secondary 53C42, 30F10. 
the family. Double solutions seem to be rare, except for genus zero or non-orientable cases (see [9] and [10]).

The present work shows that among complete orientable minimal surfaces in $\boldsymbol{R}^{3}$ with finite total curvature and positive genus, the symmetry group and the conformal type together are still insufficient to characterize the surface. The examples constructed herein are described as "birdcage-catenoids". The reason for this name comes from the fact that these surfaces are minimal immersions in $\boldsymbol{R}^{3}$ of punctured compact Riemann surfaces called "birdcages" (see [4], [5] or [6]). For $x>1$ we describe all "birdcage" Riemann surfaces of genus $n-1, n \geq 2$, as follows:

$$
M_{x}=\left\{(h, w) \in(C \cup \infty)^{2} \mid w^{n}=\frac{h+x}{h-x} \cdot \frac{h-1}{h+1}\right\} .
$$

They form a continuous family parametrized by $x$, while $w$ and $h$ are meromorphic functions on $M_{x}$. The construction of the "birdcage-catenoids" here is indirectly based on the Costa surface. As a matter of fact, the Costa surface first inspired Hoffman and Meeks for the construction of embedded minimal surfaces in $\boldsymbol{R}^{3}$ (see Figure 2(b) and [5]). For every $n \geq 3$, we can think of the "birdcage" of genus $n-1$ as the $M_{n}$-Costa-Hoffman-Meeks surface with each end compactified to a point. See the $n=3$ case in Figure 1.

In this paper we prove the following result

THEOREM 1.1. For every integer $n \geq 2$ there exist two one-parameter families of complete orientable minimal surfaces in $\boldsymbol{R}^{3}$ of finite total curvature such that, for any member of these families the following hold:

(a) The surface is conformally equivalent to a "birdcage" of genus $n-1$, punctured at the points $h^{-1}(\{-1,0,1\})$. All of its ends are of catenoidal type.

(b) The surface can be positioned to have its symmetry group generated by reflections in the planes $x_{3}=0$ and $\sin (k \pi / n) \cdot x_{1}=\cos (k \pi / n) \cdot x_{2}, k=1,2, \ldots, n$.

(c) In this same position, the surface has exactly 2 vertical ends and $n$ horizontal ends.

Moreover, there exists a $\kappa_{n}>1$ such that every $x \in\left(1, \kappa_{n}\right)$ determines one surface from each family. Both surfaces have the same symmetry group and conformal type, but they are not congruent.

REMARK. The case $n=2$ generalizes the classical toroidal four-noid (see Figure 4), of which the symmetry group includes reflection in the planes $x_{1}=x_{3}$ and $x_{1}=-x_{3}$. We do have two families of rectangular toroidal four-noids, which are included in Theorem 1.1. A $90^{\circ}$-rotation around the $x_{2}$-axis does not bring one member into the member with the same conformal type in the other family. We shall prove this fact at the end of Section 6.

The "birdcage-catenoids" were found during my doctorate in Germany, which was supported by DAAD-Deutscher Akademischer Austausch Dienst, and CAPES-Coordenação de Aperfeiçoamento de Pessoal de Nível Superior. My adviser was Professor Hermann Karcher at the University of Bonn, Germany. I thank him for his dedication, which greatly helped in the realization of my works (see [15]). 


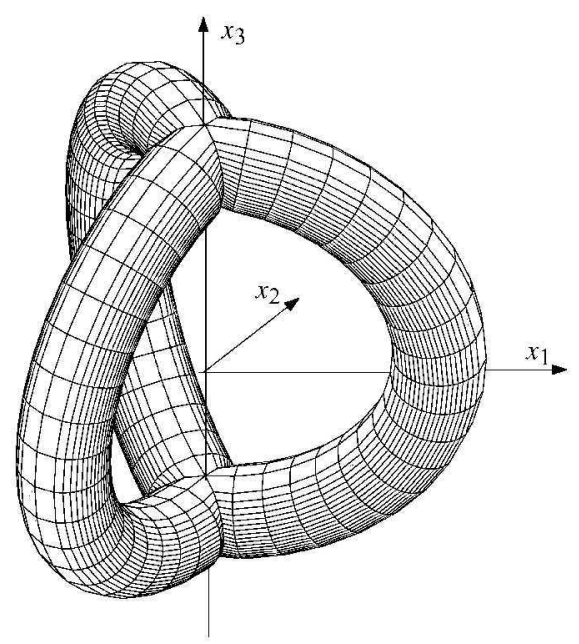

FIGURE 1. A "birdcage" of genus 2.

2. Background. In this section we state some well-known theorems on minimal surfaces. For details we refer the reader to [8], [12] and [14]. In this paper all surfaces are assumed to be regular.

THEOREM 2.1 (Weierstrass representation). Let $R$ be a Riemann surface. Let $d H$ be a meromorphic 1-form on $R$ and $g: R \rightarrow \hat{C}:=C \cup \infty$ a meromorphic function. Then $X: R \rightarrow R^{3}$ given by

$$
X(p):=\operatorname{Re} \int^{p}\left(\phi_{1}, \phi_{2}, \phi_{3}\right),
$$

where $\left(\phi_{1}, \phi_{2}, \phi_{3}\right):=(1 / 2)\left(g-g^{-1}, i\left(g+g^{-1}\right), 2\right) d H$, is a conformal regular minimal immersion provided the poles and zeros of $g$ coincide with the zeros of $\mathrm{H} H$. Conversely, every regular conformal minimal immersion $X: R \rightarrow R^{3}$ can be expressed in this form for some meromorphic function $g$ and meromorphic 1-form $d H$.

DEFINITION 2.1. The pair $(g, d H)$ is called the Weierstrass data on $R$ of the minimal immersion $X: R \rightarrow S=X(R) \subset R^{3}$.

Definition 2.2. A complete, orientable minimal surface $S$ is said to be algebraic if it admits a Weierstrass representation such that $R=\bar{R}-\left\{p_{1}, \ldots, p_{r}\right\}$, where $\bar{R}$ is compact and both $g$ and $d H$ extend meromorphically to $\bar{R}$.

DEFINITION 2.3. An end of $S$ is the image of a punctured neighbourhood $V_{p}$ of a point $p \in\left\{p_{1}, \ldots, p_{r}\right\}$ such that $\left(\left\{p_{1}, \ldots, p_{r}\right\}-p\right) \cap \bar{V}_{p}=\emptyset$. The end is embedded if this image is embedded for a sufficiently small neighbourhood of $p$. 


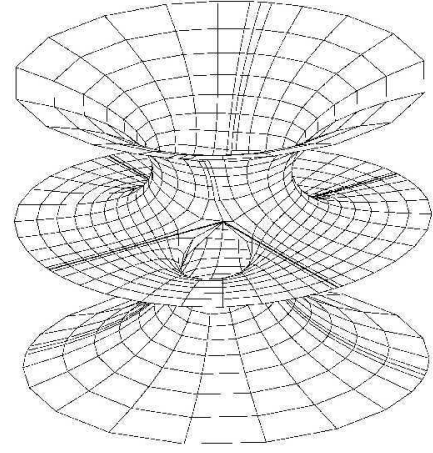

(a)

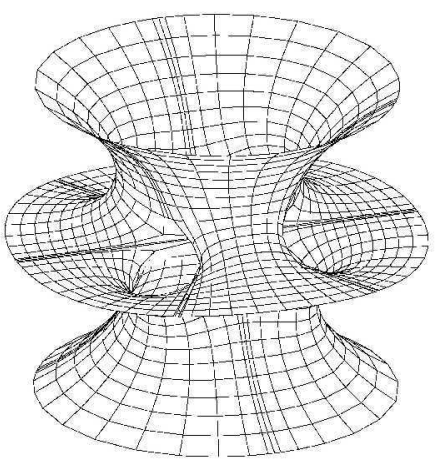

(b)

FIGURE 2. (a) The Costa surface, (b) the $M_{3}$-Costa-Hoffman-Meeks surface.

THEOREM 2.2. Let $S$ be an algebraic minimal surface of which the genus of $\bar{R}$ is $k$ and the number of ends is $r$ (all of them embedded). Then

$$
\operatorname{deg}(g)=k+r-1 .
$$

REMARK. The function $g$ is the stereographic projection of the Gauss map $N: R \rightarrow$ $S^{2}$ of the minimal immersion $X$. This minimal immersion is well-defined in $\boldsymbol{R}^{3}$ provided that

$$
\operatorname{Re} \oint\left(g-g^{-1}, i\left(g+g^{-1}\right), 2\right) d H=0
$$

for every closed path in $R$. The function $g$ is a covering map of $\hat{C}$ and the total curvature of $S$ is $-4 \pi \operatorname{deg}(g)$.

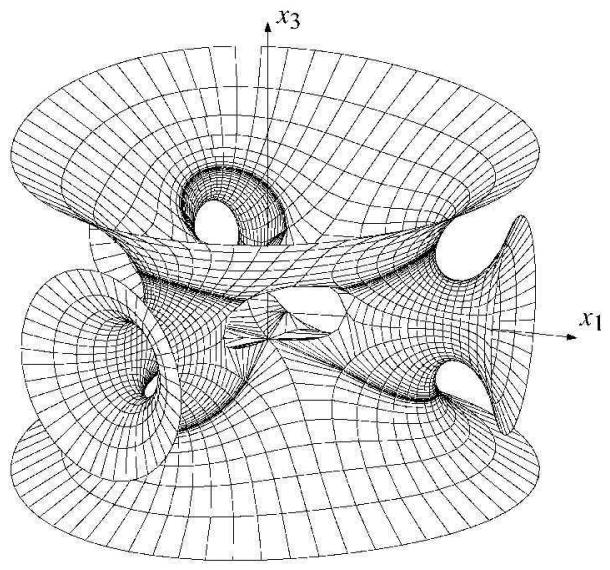

FIGURE 3. A "birdcage-catenoid" of genus 2. 
3. The involutions of the surface $M_{\chi}$. Here we summarize important involutions of $M_{x}$ related to the symmetries of $S$ in $\boldsymbol{R}^{3}$. The surface $M_{x}$ is hyperelliptic, but this property will not lead to a symmetry of $S$. Figure 5 illustrates the paths $I$ to $I V$ as fixed point sets of involutions summarized in Table (2).

(2)

\begin{tabular}{|c|c|c|c|}
\hline symmetry & involution & $h \in$ & $w \in$ \\
\hline \hline hyperelliptic & $(h, w) \rightarrow(-x / h, w)$ & $\{-i \sqrt{x}, i \sqrt{x}\}$ & $\hat{\boldsymbol{C}}$ \\
\hline$I$ & $(h, w) \rightarrow\left(\bar{h}, e^{-2 \pi i / n} \bar{w}\right)$ & {$[1, x]$} & $e^{-i \pi / n}[0, \infty]$ \\
\hline$I I$ & $(h, w) \rightarrow(\bar{h}, \bar{w})$ & {$[x, \infty]$} & {$[1, \infty]$} \\
\hline$I I I$ & $(h, w) \rightarrow(-\bar{h}, 1 / \bar{w})$ & $i \boldsymbol{R}$ & $S^{1}$ \\
\hline$I V$ & $(h, w) \rightarrow(\bar{h}, \bar{w})$ & {$[0,1]$} & {$[0,1]$} \\
\hline
\end{tabular}

4. The Gauss map on $S$ and the function $g$. We are supposing that Figure 3 represents a surface $S$ in $R^{3}$ given by a minimal immersion $X: R \rightarrow S$, where $R:=M_{x}-$ $h^{-1}(\{0, \pm 1\})$. Hence, $X$ is given by Weierstrass data $(g, d H)$ on $M_{x}$. The differential $d H$ on $M_{x}$ will be described according to the regular points and the type of ends we want the surface to have. We shall analyse this differential in the next section.

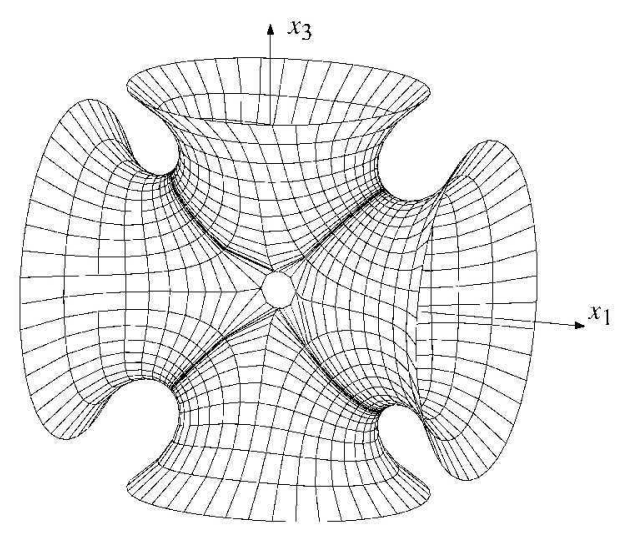

FIGURE 4. The classical toroidal four-noid.

From Figure 5, one sees that $h$ is like a "height function", in the sense that $h=+1$ at the top of $M_{x}, h=-1$ at the bottom, $h=+x$ is the upper saddle of $M_{x}, h=-x$ is the lower saddle, and the middle is exactly where $h$ is pure imaginary. This function is the same as the one used in [18], and the construction of "birdcage-catenoids" was, in fact, inspired in this work of Wohlgemuth. There the author added handles to the catenoid. From Figure 3, one can see the "birdcage-catenoids" of genus $n-1$ like $(n+2)$-noids, to which $n$ handles are added towards the interior and meet to form the saddles. 


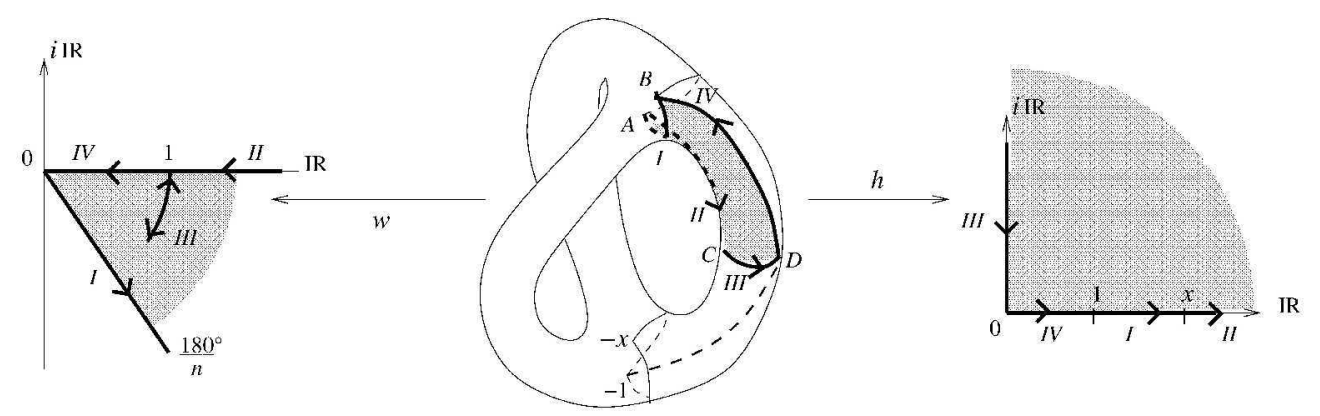

FIGURE 5. A scheme of the surface $M_{x}$ and the functions $h$ and $w$ on it.

Let us consider the surface $S$ represented in Figure 3, and its unitary normal vector. At the top catenoidal end of $S$, the vector is vertical, and we consider that it points downwards. The top catenoid of $S$ is related to the point $B \in M_{x}$, as shown in Figure 5. Hence we take $g(B)=0$. Based on Figures 3 and 5 , we realize that there is another point on path $I V$, where the corresponding normal vector on $S$ will be vertical as well. But then, it will point upwards. Hence, for a certain value $y \in(0,1)$, we have that $h=y$ implies $g=\infty$.

At the upper saddle of $S$, which corresponds to $A \in M_{x}$, the unitary normal vector is vertical and points downwards. Due to the $\left(360^{\circ} / n\right)$-rotational symmetry of $S$ around the $x_{3}$ axis, the vector has a branch of order $n-2$ at this saddle. Therefore, $g$ assumes the value 0 at $A$ with branch order $n-2$. Due to the horizontal reflectional symmetry of $S$ with respect to the plane $x_{3}=0$, it is easy to localize the other poles and zeros of $g$. They are all represented in Figure 6(d).

For a complete minimal surface of finite total curvature, genus $n-1$ and $n+2$ embedded ends we have

$$
\operatorname{deg}(g)=n-1+\frac{n+2}{2}-1+\frac{n+2}{2}=2 n,
$$

which is consistent with our analysis of $g$. Now, based on Figure 6(d), it is easy to write down the following equation:

$$
g=\frac{h+y}{h-y} \cdot \frac{x-h}{x+h} \cdot w .
$$

Of course, both sides of (3) are a priori just proportional. However, since the unitary normal vector on $S$ is horizontal at the corresponding path $I I I$ on $M_{x}$, and it points at the opposite sense of the $x_{1}$-axis at $C$, we have $g(C)=-1$. Therefore, from (3) we have that $h=\infty$ implies $g=-1 \cdot \sqrt[n+1]{1}$. Moreover, it is easy to verify the consistence of (3) regarding the expected behaviour of $g$ on paths $I$ through $I V$, and on the image of these paths under the involutions of $M_{x}$. 
5. The height differential $d H$ on $M_{x}$. In this section we are going to write explicitly the meromorphic differential $d H$ on $M_{x}$ in terms of the function $h$ and the exact differential $d h$. First of all, we need to localize the poles and zeros of $d H$ under the assumption that $S$ is the image of a minimal immersion $X: R \hookrightarrow R^{3}$, with prescribed characteristics.

Based on Figures 3 and $6(\mathrm{~b})$ one sees that, at the vertical catenoidal ends of $S$, we have $g=0$ (top) and $g=\infty$ (bottom). Hence $d H$ has a simple pole at these points. At the horizontal catenoidal ends of $S, g$ is finite and different from zero. Hence $d H$ has a pole of order 2 at these points. We recall that $S$ is supposed to be regular. We saw that at the saddles $g=0$ (top) or $g=\infty$ (bottom), both are of order $n$. Consequently, $d H$ has a zero of order $n$ at each of these saddles.

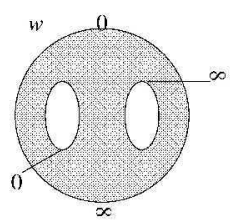

(a)

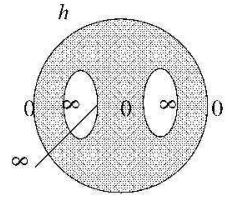

(b)

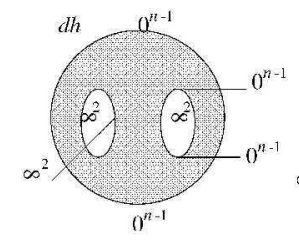

(c)

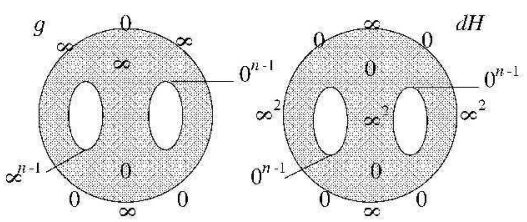

(e)

FIGURE 6. (a) Poles and zeros of $w$, (b) of $h$, (c) of $d h$, (d) of $g$, (e) of $d H$.

There are other points where $d H$ must be zero. They were discussed in the last section and correspond to $h= \pm y$, where $y \in(0,1)$. The unitary normal vector on $S$ is vertical at these points, and since we want $S$ to be regular, $d H$ must be zero there. Let us see now if this last assertion concludes our analysis about the poles and zeros of $d H$, which is summarized in Figure 6(e). We first recall that

$$
\operatorname{deg}(d H)=-\chi\left(M_{x}\right)=2(n-2),
$$

which is consistent with Figure 6(e). Moreover, we know that the poles of $d H$ are taken only at the ends of $S$, and their orders agree with the expected values of $g$ assumed there. Thus, $d H$ has exactly $2(n+1)$ poles and from (4) it must have exactly $2(2 n-1)$ zeros on $M_{x}$ (including their multiplicities). Hence Figure $6(\mathrm{e})$ is consistent with the complete analysis of poles and zeros of $d H$.

Now, from Figures 6(b-e), it is easy to write down an explicit formula for the differential $d H$ :

$$
d H=\frac{h^{2}-y^{2}}{h^{2}} \cdot \frac{d h}{1-h^{2}} .
$$

Of course, both sides of (5) are a priori just proportional. But along the path $I I I$, represented in Figure 3, the third coordinate of $S$ is expected to be constant. In other words, the value $x_{3}=\operatorname{Re} \int_{I I I^{\prime}} d H$ must be the same, for any path $I I I^{\prime} \subset I I$. Therefore, $\operatorname{Re}(d H)$ must be zero on this path, and then both sides of (5) are equal up to a non-zero real proportional constant. We choose this constant to be 1 , for another value would just rescale the surface $S$. 
At this point we remark that (3) and (5) lead to concrete Weierstrass data $(g, d H)$ on the surface $M_{x}$ defined in (1). Now the surface $S$ represented in Figure 3 can be explicitly defined as the minimal immersion of $R=M_{x}-h^{-1}(\{0, \pm 1\})$ in $\boldsymbol{R}^{3}$, given by the Weierstrass data (3) and (5). However, we must verify if this definition of $S$ is consistent with Figure 3. First of all, $S$ must have the expected symmetry curves supposed at the beginning. Afterwards, we must prove that $S$ has no periods, as Figure 3 suggests. This second task will be subject of the next section. Now we are going to verify the symmetries of $S$.

From (3) and (5) one can analyse the behaviour of $g$ and $d H$ on the paths $I$ through $I V$ indicated in Figure 3. This analysis is summarized in Table (6). From Table (6) it is easy to see that the paths $I$ through $I V$ are geodesics of $S$. Since $(d g / g) \cdot d H \in R$ on any of these paths, the geodesics are in fact reflectional symmetry curves of $S$ (see [8]).

\begin{tabular}{|c|c|c|c|}
\hline symmetry & $h \in$ & $g \in$ & $d H \in$ \\
\hline \hline$I$ & $(1, x)$ & $e^{-i \pi / n}(0, \infty)$ & $\boldsymbol{R}$ \\
\hline$I I$ & $(x, \infty)$ & $(-1,0)$ & $\boldsymbol{R}$ \\
\hline$I I I$ & $i \boldsymbol{R}$ & $S^{1}$ & $i \boldsymbol{R}$ \\
\hline$I V$ & $(0, y)$ & $(-\infty,-1)$ & $\boldsymbol{R}$ \\
\hline$I V$ & $(y, 1)$ & $(0, \infty)$ & $\boldsymbol{R}$ \\
\hline
\end{tabular}

6. Solution of the period problems. By summing up our conclusions mentioned above, we have that (1) defines a compact Riemann surface $M_{x}$ of genus $n-1$, with Weierstrass data (3) and (5) on it, and the corresponding image $S$ of the minimal immersion $X: R \hookrightarrow R^{3}$ has the symmetries that Figure 3 suggests. What we do not know yet is if $S$ has no periods, as indicated in Figure 3 .

We recall that $X(p):=\operatorname{Re} \int_{p_{0}}^{p}\left(\phi_{1}, \phi_{2}, \phi_{3}\right)$ for any $p \in R$ and a fixed $p_{0} \in R$. Therefore, if $\oint\left(\phi_{1}, \phi_{2}, \phi_{3}\right)$ is pure imaginary for every closed curve on $R$, then the period problems will be solved. Let us begin with the analysis of integrals on closed curves around each puncture of $R$. Due to the symmetries of $S$, which were just proved in the last section, it suffices to consider only the points $B$ and $D$ (see Figure 5). The point $B$ is the intersection of paths $I$ and $I V$. They are planar symmetry curves on $S$. Therefore, a small curve on $M_{x}$ around $B$ is, after homology, invariant under reflections in $I$ and $I V$. By virtue of this, the period vector $\operatorname{Re} \oint\left(\phi_{1}, \phi_{2}, \phi_{3}\right)$ is perpendicular to the planes in $\boldsymbol{R}^{3}$, in which the images of $I$ and $I V$ are contained. But (3) implies that these planes are not parallel, because $g$ is the stereographic projection of the unitary normal vector on $S$. Since the period vector $\operatorname{Re} \oint\left(\phi_{1}, \phi_{2}, \phi_{3}\right)$ must be perpendicular to both planes, we conclude that it is zero.

For a small curve around $D=I I I \cap I V$, we apply the same argument as above and conclude that the period vector will be also zero in this case. Now we must consider curves which are generators for the first homology group of $M_{x}$. It is enough to analyse only two of these curves, and if their periods are zero, the symmetries of $S$ will then imply that the whole 


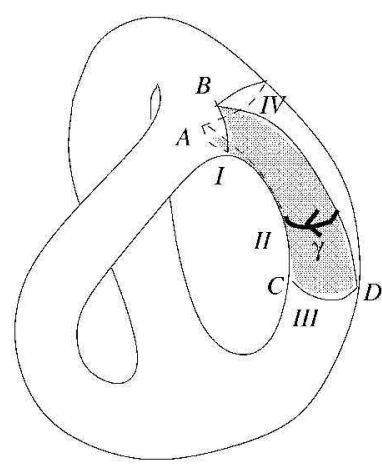

FIGURE 7. The surface $M_{x}$ and the curve $\gamma$ on it.

surface has no periods. One of these two curves is a connected component of the lifting of $h \circ I I$ by the function $h$. From Figure 5, on sees that this lifting is crossed by paths $I$ and $I I I$. After applying the same argument as above, we shall have that the period vector is also zero in this case, since it must be perpendicular to both planes in which the images of $I$ and $I I I$ are contained. It remains to analyse the other closed curve, which can be considered as the curve $\gamma$ represented in Figure 7, together with its image under reflection in path $I I$ or $I V$. By the same way, this will also imply that the period vector on the closed curve will be perpendicular to the plane $x_{2}=0$. Therefore, the only remaining task is the proof of $\operatorname{Re} \int_{\gamma} \phi_{2}=0$.

In this section it will be shown that, under certain conditions, one really has $\operatorname{Re} \int_{\gamma} \phi_{2}=$ 0 . We are working with two different real variables, $x$ and $y$, which are present in (3) and (5). Nevertheless, only the variable $x$ appears in (1), which defines our compact Riemann surface $M_{x}$. We are going to prove the following proposition.

PROPOSITION 6.1. For each natural $n \geq 2$ the following holds:

(a) There exists a unique $\kappa_{n}>1$ such that for every $x \in\left(1, \kappa_{n}\right]$ the period problem has exactly two solutions $y_{1}(x)$ and $y_{2}(x)$. For $x>\kappa_{n}$, the period problem is not solvable.

(b) The functions $y_{1}$ and $y_{2}$ are increasing and decreasing with $x$, respectively. Both are continuous and $y_{1} \leq y_{2}$, where the equality holds only for $x=\kappa_{n}$.

(c) $\lim _{x \rightarrow 1} y_{1}(x)=0$ and $\lim _{x \rightarrow 1} y_{2}(x)=1$.

The proof of Proposition 6.1 requires some preliminaries. First of all, define

$$
P(n, x, y)=-2 \operatorname{Re} \int_{\gamma} \phi_{2} .
$$

We recall that paths $I$ through $I V$ form the border of a hyperbolic quadrilateral on $M_{x}$ (see Figure 5). The interior of this quadrilateral is brought to the quadrant $\{z: 0<\operatorname{Arg}(z)<\pi / 2\}$ of $\hat{C}$. Under the Möbius transformations $(x-h) /(x+h),(1-h) /(1+h)$ and their inverses, this quadrant is always brought to open regions of $\hat{C}$ which exclude the real negative semi-axis. Therefore, the branch of the $n$-th root given by $\sqrt[n]{e^{i t}}:=e^{i t / n}$ is well-defined and continuous 
on these regions. Hence, we fix this branch and define

$$
F(x, h):=\left(\frac{x-h}{x+h}\right)^{1-1 / n} \cdot\left(\frac{1-h}{1+h}\right)^{1 / n} .
$$

By using (3), (5) and $\phi_{2}=(i / 2)\left(g+g^{-1}\right) d h$, we can rewrite (7) as the parabola

$$
P=a y^{2}+b y+c,
$$

where

$$
\begin{gathered}
a(n, x):=\operatorname{Re} \int_{\gamma} \frac{\left(F+F^{-1}\right) d h}{i h^{2}\left(1-h^{2}\right)}, \\
b(n, x):=2 \operatorname{Re} \int_{\gamma} \frac{\left(F-F^{-1}\right) d h}{i h\left(1-h^{2}\right)}, \quad \text { and } \\
c(n, x):=\operatorname{Re} \int_{\gamma} \frac{\left(F+F^{-1}\right) d h}{i\left(1-h^{2}\right)} .
\end{gathered}
$$

In the proof of Proposition 6.1 we analyse the case $x \rightarrow \infty$. To this end, the following definitions will be helpful:

$$
F(\infty, h):=\left(\frac{1-h}{1+h}\right)^{1 / n},
$$

and $a(n, \infty), b(n, \infty), c(n, \infty)$ and $P(n, \infty, y)$ defined as in (9) through (12).

Now we are going to remark two important results about the coefficients of the parabola (9), which are technical lemmas that we prove in [16] and in the Appendix (Section 7):

LEMMA 6.1. The functions $a, b$ and $c$ defined above satisfy the following properties:
(a) $b$ is negative and increasing with $x$.
(b) $0<-b<2 a$ for every $x>1$.
(c) a is positive and decreasing with $x$.
(d) $c$ is positive and increasing with $x$.
(e) $a, b$ and c are continuous at $x=1$ and $\left.c\right|_{x=1}=1+(b+c) /\left.a\right|_{x=1}=0$.
(f) The function $a+b+c$ is positive and increasing with $x$.

Lemma 6.2. The functions $a, b$ and $c$, defined by (10) through (12), are continuous at $x=\infty$. Moreover, $P(n, \infty, y)>0$ for every $y \in(0,1)$.

At this point we are ready to present

Proof of Proposition 6.1. We recall (9) and consider the normalized parabola $\tilde{P}:=a^{-1} P$ for $y \in[0,1]$. The vertex of $\tilde{P}$ is always in $[0,1] \times \boldsymbol{R}$, by (b) of Lemma 6.1. From (a), (c), (d), (e) and (f) of Lemma 6.1, we conclude that the function $1+(b+c) / a$ is positive and increasing with $x$. The same holds for $c / a$. Since $\tilde{P}$ crosses the axes $\{0\} \times \boldsymbol{R}$ and $\{1\} \times \boldsymbol{R}$ at $c / a$ and $1+(b+c) / a$, respectively, we obtain the following:

1. The discriminant $\Delta:=(b / a)^{2}-4 c / a$ is positive if and only if $\tilde{P}=0$ for exactly two different values $y=y_{1}$ and $y=y_{2}$ in the interval $(0,1)$, where $y_{1}<y_{2}$. 
2. $\Delta=0$ if and only if $\tilde{P}$ has a double zero at some $y \in(0,1)$.

3. $\Delta<0$ if and only if $\tilde{P}$ has no zeros.

From (e) of Lemma 6.1 it follows that $P(n, 1,0)=P(n, 1,1)=0$. Since $\Delta$ is continuous, the above-mentioned functions $y_{1}(x)$ and $y_{2}(x)$ are also continuous where they are defined. Moreover, $\lim _{x \rightarrow 1} y_{1}(x)=0$ and $\lim _{x \rightarrow 1} y_{2}(x)=1$. We are going to prove that $y_{1}$ and $y_{2}$ are increasing and decreasing with $x$, respectively. We denote by a prime differentiation with respect to $x$. Now define $\tilde{b}:=b / a$ and $\tilde{c}:=c / a$. Therefore,

$$
\Delta^{\prime}=2 \tilde{b} \tilde{b}^{\prime}-4 \tilde{c}^{\prime} \text {. }
$$

We can rewrite (13) as follows:

$$
\Delta^{\prime}=2 \tilde{b}\left(\tilde{b}^{\prime}+\tilde{c}^{\prime}\right)-(2 \tilde{b}+4) \tilde{c}^{\prime} .
$$

From (c), (d) and (f) of Lemma 6.1 we have

$$
\tilde{c}^{\prime}>0 \text { and }(\tilde{b}+\tilde{c})^{\prime}>0 .
$$

From (a), (b) and (c) of Lemma 6.1 we have

$$
-(2 \tilde{b}+4)<0 \text { and } \tilde{b}<0 .
$$

By applying (15) and (16) to (14), it follows that $\Delta^{\prime}<0$. Since $y_{1}=-\tilde{b} / 2-\sqrt{\Delta}$ and $y_{2}=-\tilde{b} / 2+\sqrt{\Delta}$, the functions $y_{1}$ and $y_{2}$ are both continuous, increasing and decreasing with $x$, respectively, as far as they are real.

The only remaining task is to show that there exists a positive $\kappa_{n}>1$ such that $\Delta\left(\kappa_{n}\right)=$ 0. This is an immediate consequence of Lemma 6.2.

From Proposition 6.1 it follows that the "birdcage-catenoids" corresponding to $y_{1}(x)$ and $y_{2}(x)$ are not congruent for any $x \in\left(1, \kappa_{n}\right)$. First of all, we compute the logarithmic growth of their vertical ends. According to [4, pp. 21-22], the logarithmic growth is minus the residue of $d H$ at $h=1$. We easily compute them as $\left(1-y_{1}^{2}\right) / 2$ and $\left(1-y_{2}^{2}\right) / 2$ for each surface. After rescaling both surfaces in order to get them with the same vertical logarithmic growth, we then compute the change $\delta x_{3}\left(y_{j}\right)$ of their $x_{3}$-coordinates along the curve $h(t)=t$, $x<t<\infty$ :

$$
\delta x_{3}\left(y_{1}\right)=\int_{x}^{\infty} \frac{t^{2}-y_{1}^{2}}{t^{2}\left(1-t^{2}\right)} d t \quad \text { and } \quad \delta x_{3}\left(y_{2}\right)=\frac{1-y_{1}^{2}}{1-y_{2}^{2}} \int_{x}^{\infty} \frac{t^{2}-y_{2}^{2}}{t^{2}\left(1-t^{2}\right)} d t .
$$

Since $\left(y_{2}^{2}-y_{1}^{2}\right) t>\left(y_{2}^{2}-y_{1}^{2}\right)$ implies $\left(1-y_{2}^{2}\right)\left(t^{2}-y_{1}^{2}\right)<\left(1-y_{1}^{2}\right)\left(t^{2}-y_{2}^{2}\right)$, we see that $\delta x_{3}\left(y_{1}\right)<\delta x_{3}\left(y_{2}\right)$. Hence, the two surfaces cannot be congruent.

This argument is not enough for $n=2$, because in this case we have the same number of vertical and horizontal ends. Moreover, $g$ becomes unbranched at $h= \pm x$. Hence, up to rescaling, the two solutions from Proposition 6.1 could differ by a rigid motion in $\boldsymbol{R}^{3}$. Now we prove that this is possible only when $y_{1}=y_{2}$. Let us find the values of $h$ that give $g= \pm i$. Take $y=y_{1}$. From (1) and (3) an easy computation gives us

$$
h^{4}+\left[x-2 y_{1}(x+1)+y_{1}^{2}\right] h^{2}+x y_{1}^{2}=0,
$$


with a total of four roots, as expected. Of course, for $y=y_{2}$ we get

$$
h^{4}+\left[x-2 y_{2}(x+1)+y_{2}^{2}\right] h^{2}+x y_{2}^{2}=0 .
$$

Suppose that the surfaces associated to $y_{1}$ and $y_{2}$ are rescaled in such a way that a $90^{\circ}$ rotation around the $x_{2}$-axis brings one into the other. The set of points in the torus where $g= \pm i$ remains invariant and lie on a circumference in $\boldsymbol{R}^{3}$. Therefore, both fourth-order equations must have the same roots. In particular, $x y_{1}^{2}=x y_{2}^{2}$, which implies that $y_{1}=y_{2}$ and $x=\kappa_{2}$. This must be the classical toroidal four-noid, because the $90^{\circ}$-rotation around the $x_{2}-$ axis, followed by a reflection in $x_{3}=0$, gives a reflection in the plane $x_{1}=x_{3}$. Analogously, we obtain the reflection in the plane $x_{1}=-x_{3}$.

7. Appendix. The proof of Lemma 6.1 can be found in [16]. This last section is devoted to the proof of Lemma 6.2.

ProOF OF LEMMA 6.2. We are going to divide the demonstration in three steps.

Step I. The function $P(n, x, y)$ is continuous at $x=\infty$.

First, we need to make some estimates. Consider $0<\varepsilon<1$. Then,

$$
|h| \leq \frac{\varepsilon}{2+\varepsilon} \cdot x \Rightarrow\left\{\begin{array}{l}
\text { (i) } 1-\varepsilon \leq\left|\frac{x-h}{x+h}\right| \leq 1+\varepsilon, \\
\text { (ii) } \frac{1}{1+\varepsilon} \leq\left|\frac{x+h}{x-h}\right| \leq \frac{1}{1-\varepsilon} .
\end{array}\right.
$$

Since $1-\varepsilon<\sqrt[n]{1-\varepsilon}<\sqrt[n]{1+\varepsilon}<1+\varepsilon$, from (i) and (ii) we obtain

$$
1-\frac{2 \varepsilon}{1-\varepsilon}<1-\frac{2 \varepsilon}{1+\varepsilon}<\frac{1-\varepsilon}{\sqrt[n]{1+\varepsilon}} \leq\left|\frac{x-h}{x+h}\right|^{(n-1) / n} \leq \frac{1+\varepsilon}{\sqrt[n]{1-\varepsilon}}<1+\frac{2 \varepsilon}{1-\varepsilon},
$$

and hence

$$
1-\frac{2 \varepsilon}{1-\varepsilon}<1-\frac{2 \varepsilon}{1+\varepsilon} \leq\left|\frac{x+h}{x-h}\right|^{(n-1) / n} \leq 1+\frac{2 \varepsilon}{1-\varepsilon} .
$$

For $\eta:=2 \varepsilon /(1-\varepsilon)$ we conclude that

$$
\left|\left(\frac{x-h}{x+h}\right)^{(n-1) / n}-1\right|<\eta \quad \text { and }\left|\left(\frac{x+h}{x-h}\right)^{(n-1) / n}-1\right|<\eta .
$$

Let $M=M(x, \varepsilon):=\varepsilon x /(2+\varepsilon)$. We are going to consider $h \circ \gamma(t)=y+i t$, for $0<t<\infty$, and split up the curve $\gamma$ in such a way that $\gamma=\gamma_{1} \cup \gamma_{2}$, where $\left|h \circ \gamma_{1}\right| \leq M$ and $\left|h \circ \gamma_{2}\right| \geq M$.

The Möbius transformations $(x-h) /(x+h)$ and $(x+h) /(x-h)$ bring the curve $h \circ \gamma$ to half circumferences in $\hat{C}$ whose radii are smaller than $(x+y) /(x-y)$. Moreover, for $x \geq 3 y$ the inequality $(x+y) /(x-y) \leq 2$ holds. Hence, since $\left|h \circ \gamma_{2}\right| \geq M$, we have

$$
\left|\left(\frac{x-h}{x+h}\right)^{(n-1) / n}-1\right|<2^{(n-1) / n}+1<3 \quad \text { on } h \circ \gamma_{2}
$$




$$
\left|\left(\frac{x+h}{x-h}\right)^{(n-1) / n}-1\right|<3 \quad \text { on } h \circ \gamma_{2} .
$$

Similarly, it is easy to conclude that

$$
\left|\frac{1-h}{1+h}\right|^{1 / n}<\left(\frac{1+y}{1-y}\right)^{1 / n}<\frac{1+y}{1-y} \text { on } h \circ \gamma .
$$

From (17) through (19) we conclude that

$$
|P(n, x, y)-P(n, \infty, y)| \leq \eta I_{1}+3 I_{2},
$$

where

$$
I_{1}=\frac{1+y}{1-y} \int_{\gamma_{1}} \frac{\left(|h+y|^{2}+|h-y|^{2}\right)|d h|}{\left|h^{2}-h^{4}\right|}, \quad I_{2}=\frac{1+y}{1-y} \int_{\gamma_{2}} \frac{\left(|h+y|^{2}+|h-y|^{2}\right)|d h|}{\left|h^{2}-h^{4}\right|} .
$$

The quotients $(h \pm y) /(h \pm 1)$ are Möbius transformations which bring the curve $h \circ \gamma=$ $a+i t, 0 \leq t<\infty$, to half circumferences in $\hat{\boldsymbol{C}}$. Their radii are smaller than $(1+y) /(1-y)$. Hence, $|h \pm y|^{2} /\left|h^{2}-1\right| \leq(1+y)^{2} /(1-y)^{2}$ and therefore

$$
I_{1} \leq\left(\frac{1+y}{1-y}\right)^{3} \int_{h \circ \gamma_{1}} \frac{d t}{y^{2}+t^{2}}, \quad I_{2} \leq\left(\frac{1+y}{1-y}\right)^{3} \int_{h \circ \gamma_{2}} \frac{d t}{t^{2}}
$$

Now observe the following: $\left|h \circ \gamma_{1}(t)\right|=|y+i t| \leq M$ implies that $t \leq M+y$, and $\left|h \circ \gamma_{2}(t)\right|=|y+i t| \geq M$ implies that $t \geq M-y$. Because of this, if $0<\varepsilon<1, M>1$ and $x \geq 3 y$, then we have

$$
\begin{gathered}
I_{1} \leq\left(\frac{1+y}{1-y}\right)^{3} \cdot \frac{1}{y} \arctan \frac{M+y}{y} \leq \frac{(1+y)^{3} \pi}{y(1-y)^{3}}, \\
I_{2} \leq \frac{(1+y)^{3}}{(1-y)^{3}(M-y)} .
\end{gathered}
$$

By applying (21) and (22) to (20) we then get

$$
|P(n, x, y)-P(n, \infty, y)| \leq \frac{(1+y)^{3}}{y(1-y)^{3}}\left(\eta \pi+\frac{3 y}{M-y}\right) .
$$

We recall that $M=M(x, \varepsilon)=\varepsilon x /(2+\varepsilon)$ and observe the following:

$$
x>\frac{2+\varepsilon}{\varepsilon^{2}} \text { and } \varepsilon<\frac{1}{2} \text { imply }\left\{\begin{array}{l}
M>\frac{1}{\varepsilon}, \\
\eta<4 \varepsilon .
\end{array} \text { and hence } \frac{1}{M-y}<2 \varepsilon,\right.
$$

Define $k:=y^{-1}(1-y)^{-3}(1+y)^{3}(4 \pi+6 y)$ and consider $\varepsilon=\min \{1 / 3, \tilde{\varepsilon} / k\}$ for any given $\tilde{\varepsilon}>$ 0 . Finally, by applying (24) to (23), we see that for a given $\tilde{\varepsilon}>0$, there exists $N=(2+\varepsilon) / \varepsilon^{2}$ such that $x \geq N$ implies that $|P(n, x, y)-P(n, \infty, y)|<\tilde{\varepsilon}$. Hence, $P$ is continuous at $x=\infty$.

Step II. The functions $a, b$ and $c$ are continuous at $x=\infty$.

We have just proved that for any $\tilde{\varepsilon}>0$ there exists $N>0$ such that if $x \geq N$, then $|P(n, x, y)-P(n, \infty, y)|<\tilde{\varepsilon}$. Equivalently, for any sequence of positive real numbers $\left\{r_{m}\right\}$ 
such that $\lim _{m \rightarrow \infty} r_{m}=+\infty$, it follows that $\lim _{m \rightarrow \infty} P\left(n, r_{m}, y\right)=P(n, \infty, y)$. This last assertion holds if and only if $P\left(n, r_{m}, y\right)$ is a Cauchy sequence. Thus, for every $\tilde{\varepsilon}>0$ there is an $N>0$ such that if $m_{1}, m_{2} \geq N$, then

$$
\left|P\left(n, r_{m_{1}}, y\right)-P\left(n, r_{m_{2}}, y\right)\right|<\tilde{\varepsilon} .
$$

Without loss of generality, we may suppose $r_{m_{2}}>r_{m_{1}}$. From (9) and (25) we then have

$$
\left|\left(a\left(r_{m_{2}}\right)-a\left(r_{m_{1}}\right)\right) y^{2}+\left(b\left(r_{m_{2}}\right)-b\left(r_{m_{1}}\right)\right) y+\left(c\left(r_{m_{2}}\right)-c\left(r_{m_{1}}\right)\right)\right|<\tilde{\varepsilon},
$$

which can be rewritten as

$$
\begin{aligned}
& \mid\left[(a+b+c)\left(r_{m_{2}}\right)-(a+b+c)\left(r_{m_{1}}\right)\right] y^{2} \\
& \quad+\left(b\left(r_{m_{2}}\right)-b\left(r_{m_{1}}\right)\right)\left(y-y^{2}\right)+\left(c\left(r_{m_{2}}\right)-c\left(r_{m_{1}}\right)\right)\left(1-y^{2}\right) \mid<\tilde{\varepsilon} .
\end{aligned}
$$

Now apply $y=1 / 2$ to (26). From Lemma 6.1 it follows that every term of the sum inside the modulus in (26) is positive. Therefore, we have

$$
\begin{aligned}
\left|(a+b+c)\left(r_{m_{2}}\right)-(a+b+c)\left(r_{m_{1}}\right)\right| & <4 \tilde{\varepsilon}, \\
\left|b\left(r_{m_{2}}\right)-b\left(r_{m_{1}}\right)\right|<4 \tilde{\varepsilon}, \quad\left|c\left(r_{m_{2}}\right)-c\left(r_{m_{1}}\right)\right| & <\frac{3}{4} \tilde{\varepsilon} .
\end{aligned}
$$

From (27) it follows that $a\left(r_{m}\right), b\left(r_{m}\right)$ and $c\left(r_{m}\right)$ are Cauchy sequences. Of course, this conclusion does not depend on the choice of $y \in(0,1)$. Hence, we define $\hat{a}:=\lim _{m \rightarrow \infty} a\left(r_{m}\right)$, and similarly $\hat{b}$ and $\hat{c}$. Together with (25) and (26), this implies that for any $y \in \boldsymbol{R}$

$$
P(n, \infty, y)=\hat{a} y^{2}+\hat{b} y+\hat{c} .
$$

Therefore, $\hat{a}=a(\infty)$ and the analogue holds for $\hat{b}$ and $\hat{c}$. This concludes the second step of our demonstration.

Step III. $\quad P(n, \infty, y)>0$ for every $y \in(0,1)$.

Among all demonstrations in this work, the proof of this last step is the most geometrical. In the case when $x=\infty$, we are going to verify that $P(n, \infty, y)$ can be analysed on " $(\mathrm{n}+2)$ noids" (see Figure 8).

From Steps I and II, we may "simplify" the $M_{x}$ equation in (1) and work with $w^{n}=$ $(1-h) /(1+h)$. Such simplification does not correspond to a "limit surface" of the birdcage family, since the genus of $w^{n}=(1-h) /(1+h)$ is zero. For this reason, we shall use different notation. Let us first consider the following branched covering of the sphere:

$$
z^{n}=\frac{\tilde{h}-1}{\tilde{h}+1} .
$$

We want to deduce Weierstrass data $(G, d \tilde{H})$ on the sphere at (28), which lead to " $(\mathrm{n}+2)$ noids" with the same euclidean symmetries as the birdcages of genus $n-1$. For this, the arguments used to derive (3) and (5) can be analogously applied, and one obtains the following formulas:

$$
G=\frac{\tilde{h}+y}{\tilde{h}-y} \cdot z
$$




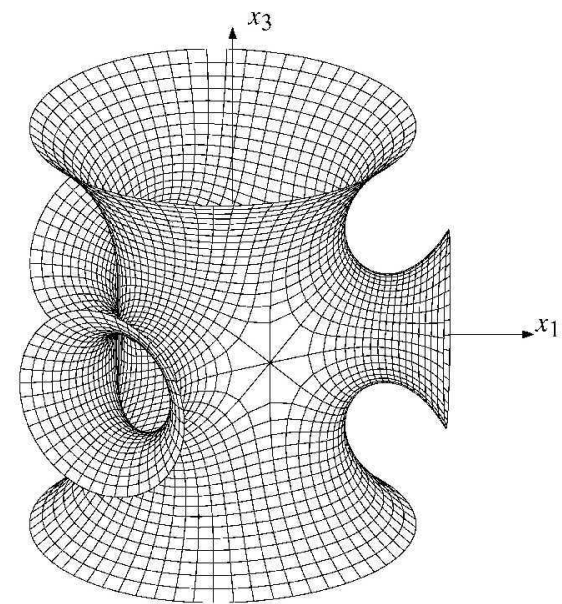

FIGURE 8. A "five-noid" with the same euclidean symmetries as the birdcages of genus 2.

$$
d \tilde{H}=\frac{\tilde{h}^{2}-y^{2}}{\tilde{h}^{2}} \cdot \frac{d \tilde{h}}{1-\tilde{h}^{2}} .
$$

From (28) we have $\tilde{h}=\left(1-z^{n}\right) /\left(1+z^{n}\right)$. If we define $\lambda:=(1+y) /(1-y)$, then a simple calculation shows that (29) and (30) are equivalent to

$$
G=z \cdot \frac{z^{n}-\lambda}{\lambda z^{n}-1}
$$

$$
d \tilde{H}=\frac{\left(z^{n}-\lambda\right)\left(\lambda^{-1}-z^{n}\right)}{z\left(z^{n}-1\right)^{2}} \cdot d z=\frac{\lambda+\lambda^{-1}-\left(z^{n}+z^{-n}\right)}{z^{n}+z^{-n}-2} \cdot \frac{d z}{z} .
$$

Of course, (32) is valid up to a proportional constant, which is $6 \lambda /\left(1+\lambda^{2}\right)$ in this case. This constant is irrelevant for our purpose. Moreover, by considering $z$ as the standard complex coordinate on $\hat{\boldsymbol{C}}$, from Figure 9 it is easy to verify (31) and (32).

Now we are going to make use of the following geometrical argument. The curves $z_{1}(t)=t, z_{2}(t)=t e^{-i \pi / n}$ and $z_{3}(t)=e^{-i t \pi / n}$ for $0 \leq t \leq 1$ lead to planar geodesics of the "(n+2)-noid". The images of $z_{1}$ and $z_{2}$ under $G$ are both straight lines which cross at $0 \in C$ and make a $\left(180^{\circ} / n\right)$-angle. Therefore, the corresponding geodesics on the " $(\mathrm{n}+2)$ noid" lie on two different vertical planes of $\boldsymbol{R}^{3}$, which are not parallel. If we position our "( $(\mathrm{n}+2)$-noid" as shown in Figure 8, we can even specify these two planes, namely, $x_{2}=0$ and $\sin (\pi / n) \cdot x_{1}=-\cos (\pi / n) \cdot x_{2}$. The third geodesic, which corresponds to the curve $z_{3}$, lies in the plane $x_{3}=0$. Figure 10 illustrates the image under $G$ of the shaded sector bordered by $z_{1}, z_{2}$ and $z_{3}$. 


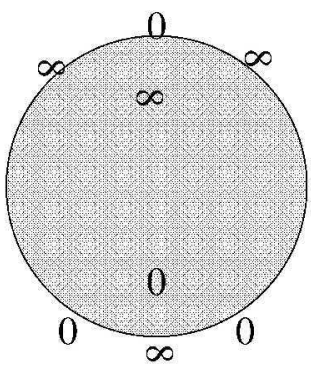

G

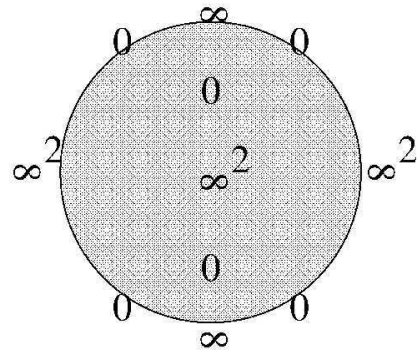

$d \tilde{H}$

FIGURE 9. The Weierstrass data $G$ and $d \tilde{H}$ for the "five-noid" on the sphere.

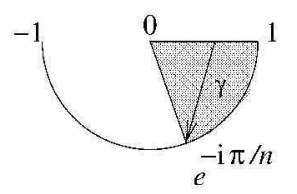

G

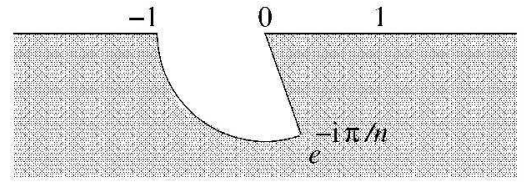

FIGURE 10. The image under $G$ of the sector bordered by $z_{1}, z_{2}$ and $z_{3}$.

An easy calculation then leads to

$$
\phi_{2}=\frac{i}{2}\left(G+G^{-1}\right) d \tilde{H}=\frac{-i}{z\left(z^{n}-1\right)^{2}}\left[\frac{z}{\lambda}\left(z^{n}-\lambda\right)^{2}+\frac{\lambda}{z}\left(z^{n}-\lambda^{-1}\right)^{2}\right] .
$$

In Figure 10, the curve $\gamma$ represents the shortest geodesic connecting $h=y$ (or equivalently, $z=\sqrt[n]{\lambda^{-1}}$ ) and $z=e^{-i \pi / n}$. This geodesic is entirely inside the circular sector, otherwise the symmetries of the " $(n+2)$-noid" could bring its pieces there, and $\gamma$ would be a piecewise shortest curve, and hence regular. Now consider

$$
x_{2}(t)=\operatorname{Re} \int_{0}^{t} \phi_{2}(\gamma(\tau)) \gamma^{\prime}(\tau) d \tau, \quad 0 \leq t \leq 1
$$

Then, $x_{2}(1)=\operatorname{Re} \int_{\gamma} \phi_{2}$ and $x_{2}^{\prime}(t)=\operatorname{Re}\left[\phi_{2}(\gamma(t)) \gamma^{\prime}(t)\right]$. An easy calculation shows that $\left.\phi_{2}\right|_{z=\sqrt[n]{\lambda^{-1}}} \in i \boldsymbol{R}_{-}^{*}$. Moreover, since $\operatorname{Im}\left\{\gamma^{\prime}(0)\right\} \in \boldsymbol{R}_{-}^{*}$, we conclude that

$$
x_{2}^{\prime}(0) \text { is negative. }
$$

Let $\Gamma$ be the composition of the minimal immersion in $\boldsymbol{R}^{3}$, given by $(G, d \tilde{H})$, with the curve $\gamma$. Then, $x_{2}^{\prime \prime}(t)$ is the second component of the normal vector $\Gamma^{\prime \prime}(t)$ on the surface, restricted to the geodesic $\Gamma$. Clearly, the modulus of this normal vector is given by $\left|\Gamma^{\prime \prime}(t)\right|$. We are going to show that $\left|\Gamma^{\prime \prime}(t)\right|$ never vanishes for $t \in(0,1)$. Let us look at the formula of 
the Gaussian curvature for minimal surfaces:

$$
K=\frac{-16}{\left(|G|+|G|^{-1}\right)^{4}}\left|\frac{d G / G}{d \tilde{H}}\right|^{2} .
$$

Based on Figures 9 and 10, one sees that neither $G$ nor $d \tilde{H}$ have poles or zeros inside the circular sector. Moreover, $d G$ has $2(n+1)$ poles in total, and since $\operatorname{deg}(d G)=-2$, the number of zeros of $d G$ must be $2 n$. If $d G$ had a zero inside the sector, the symmetries of the "(n+2)-noid" would imply that $d G$ should have at least $4 n$ zeros, which is a contradiction. Therefore, the zeros of $d G$ must be on the curves $z_{1}, z_{2}$ or $z_{3}$, and on their images under the symmetries of the " $(\mathbf{n}+2)$-noid". This implies that the Gaussian curvature of the " $(\mathbf{n}+2)$-noid" does not vanish inside the sector. Therefore, $\left|\Gamma^{\prime \prime}(t)\right|$ is always positive, for $0<t<1$.

Now consider again Figure 10. The shaded region at the right-hand side is the stereographic projection of the unitary normal on the corresponding region of the "( $(\mathrm{n}+2)$-noid". Since $\left|\Gamma^{\prime \prime}(t)\right|>0$ for $t \in(0,1)$, we conclude that the sign of $x_{2}^{\prime \prime}(t)$ is always the same for $t \in(0,1)$. Therefore,

$$
\begin{gathered}
\text { either } x_{2}^{\prime \prime}(t)<0, \quad t \in(0,1), \\
\text { or } \quad x_{2}^{\prime \prime}(t)>0, \quad t \in(0,1) .
\end{gathered}
$$

If (34) is valid, then (33) implies that $x_{2}^{\prime}(t)<0$ for $t \in(0,1)$ and consequently $P(n, \infty, y)=$ $-2 x_{2}(1)>0$ from (7). If (35) holds, then we are still able to obtain the same conclusion, namely $P(n, \infty, y)>0$, if we prove that $x_{2}^{\prime}(1) \leq 0$. In this case, $x_{2}^{\prime}$ then assumes no maximum in $(0,1)$ and hence $x_{2}^{\prime}(t)<0$ for $t \in(0,1)$, which again implies that $P(n, \infty, y)=$ $-2 x_{2}(1)>0$.

From Figure 10 one sees that $-\pi / n-\pi / 2<\operatorname{Arg}\left\{\gamma^{\prime}(1)\right\}<-\pi / n$. Moreover, an easy calculation leads to

$$
\phi_{2}(\gamma(1))=\frac{-(1+\lambda)^{2} i}{4 \lambda} \cdot\left(1+e^{2 i \pi / n}\right) .
$$

Thus, $n>3$ implies that $-\pi<\operatorname{Arg}\left\{\phi_{2}(\gamma(1)) \gamma^{\prime}(1)\right\}<-\pi / 2$ and therefore, $x_{2}^{\prime}(1) \leq 0$. For $n=2$ we have $x_{2}^{\prime}(1)=0$.

\section{REFERENCES}

[1] C. C. Chen And F. Gackstatter, Elliptische und Hyperelliptische Funktionen und vollständige Minimalflächen von Enneperschen Typ, Math. Ann. 259 (1982), 359-369.

[2] C. CosTA, Example of a complete minimal immersion in $\boldsymbol{R}^{3}$ of genus one and three embedded ends, Bol. Soc. Brasil. Mat. 15 (1984), 41-54.

[ 3 ] C. Costa, Uniqueness of minimal surfaces embedded in $\boldsymbol{R}^{3}$ with total curvature $12 \pi$, I. Differential Geom. 30 (1989), 597-618.

[4] D. HOFFMAN AND H. KARCHER, Complete embedded minimal surfaces of finite total curvature, Encyclopaedia of Math. Sci. 90, Ed. R. Osserman, 5-93, Springer-Verlag, Berlin, Heidelberg, 1997.

[ 5 ] D. HOFFMAN AND W. MEEKs, A complete embedded minimal surface in $\boldsymbol{R}^{3}$ with genus one and three ends, J. Differential. Geom. 21 (1985), 109-127. 
[6] D. Hoffman AND W. MEEks, Embedded minimal surfaces of finite topology, Ann. of Math. 131 (1990), $1-34$.

[7] A. Huber, On subharmonic functions and differential geometry in the large, Comment. Math. Helv. 32 (1957), 13-72.

[8] H. KARCHER, Construction of minimal surfaces, Surveys in Geometry, University of Tokyo (1989), 1-96, and Lecture Notes 12, SFB256, Bonn, 1989.

[9] F. J. LóPEZ, The classification of complete minimal surfaces with total curvature greater than $-12 \pi$, Trans. Amer. Math. Soc. 334 (1992), 49-74.

[10] F. J. LOPEZ AND F. MARTíN, Complete nonorientable minimal surfaces with the highest symmetry group, Amer. J. Math. 119 (1997), 55-81.

[11] F. J. LÓPEZ AND A. RoS, On embedded complete minimal surfaces of genus zero, J. Differential Geom. 33 (1991), 293-300.

[12] J. C. C. Nitsche, Lectures on minimal surfaces, Second edition, Dover, New York, 1986

[13] R. Osserman, Global properties of minimal surfaces in $E^{3}$ and $E^{n}$, Ann. of Math. (2) 80 (1964), 340-364.

[14] R. Osserman, A survey of minimal surfaces, Second edition, Dover Publications, New York, 1986.

[15] V. Ramos BATISTA, Construction of new complete minimal surfaces in $\boldsymbol{R}^{3}$ based on the Costa surface, Doctoral thesis, University of Bonn, 2000.

[16] V. RAmos BATISTA, The use of unitary functions in the behaviour analysis of elliptic integrals, Technical Report 03/03, University of Campinas, 2003. http://www.ime.unicamp.br/rel_pesq/2003/rp03-03.html.

[17] R. Schoen, Uniqueness, symmetry and embeddedness of minimal surfaces, J. Differential Geom. 18 (1983), 791-809.

[18] M. WoHL GEMUTH, Higher genus minimal surfaces by growing handles out of a catenoid, Manuscripta Math. 70 (1991), 397-428.

INSTITUTE OF MATHEMATICS

STATISTICS AND COMPUTER SCIENCES

UNIVERSITY OF CAMPINAS

P.O. BOX: 6065

13083-970 CAMPINAS-SP

BRASIL

E-mail address: valerio@ime.unicamp.br 